\title{
Comprehensive review and future perspectives on the photocatalytic hydrogen production
}

\author{
Juan Corredor, ${ }^{\mathrm{a}} \odot$ María J Rivero, $^{\mathrm{a}}$ Carmen M Rangel, ${ }^{\mathrm{b}}$ Frederic Gloaguenc \\ and Inmaculada Ortiz ${ }^{\text {a* }}$
}

\begin{abstract}
Hydrogen represents a renewable energy alternative that may positively contribute to get over the global energy crisis while at the same time reducing its environmental burden. Overcoming the challenge of reaching this potential could be helped by careful choice of hydrogen $\left(\mathrm{H}_{2}\right)$ sources. Photocatalytic generation of $\mathrm{H}_{2}$, although a minor alternative, appears to be a very good option at the time that liquid wastes are being degraded; therefore, this approach has given rise to an increasing number of interesting studies. Here, we aim to provide an integrated overview of the different photocatalytic, heterogeneous, homogeneous and hybrid systems. First, we categorize the units and mechanisms that take part in the photocatalytic process, and secondly we analyze their role and draw comparative conclusions. Thus, we analyze the role of (i) the electron source to carry out proton reduction, (ii) the proton source, which can be free protons in the medium or a proton donor compound, (iii) the catalyst nature and concentration, and (iv) the photosensitizer nature and concentration. We also provide an analysis of the influence of the solvent, especially in homogenous systems as well as the influence of pH. We provide a comparison of the photocatalytic performance, highlighting the advantages and disadvantages, of different systems. Thus, this review is, on the one hand, an update on the state of the art of photocatalytic generation of $\mathrm{H}_{2}$ from a full perspective that integrates homogeneous, heterogeneous and hybrid systems, and, on the other, a source of useful information for future research.
\end{abstract} (c) 2019 Society of Chemical Industry

Keywords: photocatalytic hydrogen production; homogeneous, heterogeneous and hybrid systems; comparative performance

\section{STATE OF THE ART OF PHOTOCATALYTIC GENERATION OF HYDROGEN}

Increased emissions of greenhouse gases to the atmosphere due to fossil fuel combustion pose a serious threat to the environment. Hydrogen $\left(\mathrm{H}_{2}\right)$-based energy comes out as a clean alternative because its direct combustion does not generate pollutants or greenhouse gases. ${ }^{1-5}$ Nowadays, $95 \%$ of $\mathrm{H}_{2}$ is obtained by steam reforming of fossil fuels, mainly methane. This process operates at high temperatures and pressures, and thus is energy-intensive. ${ }^{6-8}$ Water $\left(\mathrm{H}_{2} \mathrm{O}\right)$ electrolysis is one of the most promising $\mathrm{H}_{2}$ production alternatives, especially, for storage of surplus electricity; a variety of commercial electrolysers for large-scale applications is available on the market.,10 However, the energy consumption associated with electrolysis must be significantly reduced before this process becomes competitive at large scales. Photocatalytic generation of $\mathrm{H}_{2}$ from organic solutions or $\mathrm{H}_{2} \mathrm{O}$, although considered a minor source in terms of produced $\mathrm{H}_{2}$ volume, appears to be a complementary and renewable way for simultaneous $\mathrm{H}_{2}$ generation and wastewater remediation. ${ }^{1-16}$

In 1972, Fujishima and Honda reported the first heterogeneous photocatalytic $\mathrm{H}_{2}$ production, HETPHP, by water splitting. ${ }^{3,17} \mathrm{HET}$ PHPs are assisted by a semiconductor that acts as catalyst and light harvester. Five years later, Lehn et al. (1977) published the first homogeneous photocatalytic $\mathrm{H}_{2}$ production, HOMPHP, from triethanolamine (TEOA). ${ }^{18}$ Typical HOMPHPs involve the use of an organometallic complex as catalyst and a second organic or organometalic compound as photosensitizer. More recently, hybrid photocatalytic $\mathrm{H}_{2}$ production systems, HYBPHPs, have been developed making use of the system units of both HETPHPs and HOMPHPs, homogeneous catalysts and heterogeneous photosensitizer semiconductors. ${ }^{19}$

More recently, interesting reviews have addressed separately each of the three system categories ${ }^{20-26}$ but there is no integrated and comparative analysis of the three systems. The present review provides such an integrated overview of the photocatalytic generation of $\mathrm{H}_{2}$, and the influence of the photocatalytic units and process variables on $\mathrm{H}_{2}$ production rate and stability. This comparison has been made in terms of $\mathrm{H}_{2}$ production per unit time per gram of catalyst $\left(\mathrm{mol}_{\mathrm{H} 2} \cdot \mathrm{g}_{\mathrm{cat}}{ }^{-1} \cdot \mathrm{time}^{-1}\right)$ for HETPHPs, turnover Frequency (TOF) for HOMPHPs and HYBPHPs, and moles of $\mathrm{H}_{2}$ per mole of catalyst, turnover number (TON), as defined in Eqns (1) and (2).

The stability of the photocatalytic system, and the process efficiency are determined through the 'Quantum Yield' (QY) and 'apparent Quantum Yield' (AQY) Eqns (3) and (4), ${ }^{23-27}$ as indicators

\footnotetext{
Correspondence to: I Ortiz, Department of Chemical and Biomolecular Engineering, ETSIIT, University of Cantabria, Avda. de los Castros s/n, 39005, Santander, Spain.E-mail: inmaculada.ortiz@unican.es

a Department of Chemical and Biomolecular Engineering, ETSIIT, University of Cantabria, Santander, Spain

b Laboratório Nacional de Energia e Geologia, Lisbon, Portugal

c UMR 6521, CNRS, Université de Bretagne Occidentale, Brest, France
} 